\title{
Management of hyperthyroidism in pregnancy
}

\author{
Rashmi Aggarwal*, Pradeep Chugh
}

Department of Thyroid and Endocrine Research Institute of Nuclear Medicine and Allies Sciences, Timarpur, Delhi, India

Received: 05 December 2015

Revised: 15 December 2015

Accepted: 22 December 2015

\section{*Correspondence:}

Dr. Rashmi Aggarwal,

E-mail: drarashmi@yahoo.co.in

Copyright: (c) the author(s), publisher and licensee Medip Academy. This is an open-access article distributed under the terms of the Creative Commons Attribution Non-Commercial License, which permits unrestricted non-commercial use, distribution, and reproduction in any medium, provided the original work is properly cited.

\begin{abstract}
Hyperthyroidism in pregnancy is associated with adverse foetal, maternal and obstetrical outcome. Untreated or inadequately treated hyperthyroidism may precipitate pre eclampsia and congestive cardiac failure in mother. It also increases the risk of miscarriage, abruption placentae and premature delivery in such patients. Maintaining euthyroidism in these patients is of utmost importance. Antithyroid medications are used as first line treatment for such patients to restore euthyroid status at the earliest. Radioactive iodine is absolutely contraindicated in pregnancy and surgery often requires pre-treatment with anti thyroid medications. Two drugs are available -carbimazole and propylthiouracil. Use of carbimazole/methimazole in pregnancy is not only associated with increased incidence of scalp defect(aplasia cutis ) in the infants, but some specific congenital malformation like choanal atresia, oesophageal atresia, trachea-oesophageal fistula, patent vitello intestinal duct, omphalocele, dysmorphic facial features and growth retardation do occur. These malformations represent carbimazole /methimazole embryopathy. Due to the association of foetal teratogenicity with carbimazole /methimazole, propylthiouracil is recommended as the drug of choice in first trimester of pregnancy. However, as its use is associated with risk of hepatotoxicity, it should be changed to carbimazole/methimazole thereafter.
\end{abstract}

Keywords: Hyperthyroidism, Pregnancy, Carbimazole, Prophyl thio uracil

\section{INTRODUCTION}

Hyperthyroidism occurs in $0.1-0.4 \%$ of pregnancies and is most commonly due to Graves' disease. ${ }^{1}$ Other causes include toxic nodule, toxic multinodular goitre and thyroiditis. Diagnosis of hyperthyroidism presenting for the first time in pregnancy often becomes difficult because the symptoms and signs of hyperthyroidism like excessive sweating, palpitations, nervousness, dyspnoea, extreme fatigue and cardiac systolic murmurs can be seen in normal pregnancy. Gestational transient thyrotoxicosis is seen in women with hyperemesis gravidarum, and is due to high concentrations of circulating human chorionic gonadotropin which directly stimulates the maternal thyroid gland. Gestational transient thyrotoxicosis is usually observed in the first trimester and is frequently associated with severe vomiting. Hyperthyroidism in pregnancy is associated with adverse foetal, maternal and obstetrical outcome (Table 1). Untreated or inadequately treated hyperthyroidism may precipitate pre eclampsia and congestive cardiac failure in mother. ${ }^{3,4}$ It also increases the risk of miscarriage, abruption placentae and premature delivery in such patients. Thyroid storm can be precipitated in severe cases leading on to increased morbidity and mortality. Maintaining euthyroidism in these patients is of utmost importance. Several studies have shown that treating hyperthyroid patients in pregnancy improves the outcome of pregnancy. ${ }^{5}$ Antithyroid medications are used as first line treatment for such patients to restore euthyroid status at the earliest. 
Radioactive iodine is absolutely contraindicated in pregnancy and surgery often requires pre-treatment with anti-thyroid medications.

Table 1: Consequences of untreated Graves' disease in pregnancy.

\begin{tabular}{|l|}
\hline Maternal complications \\
- Miscarriage \\
- Placenta abruption \\
- Preterm delivery \\
- Congestive cardiac failure \\
Fetal complications \\
- $\quad$ Preonatal hyperthyroidism \\
- Prematurity \\
- Intra uterine growth retardation \\
\hline
\end{tabular}

\section{Diagnosis of Graves’ disease in pregnancy}

Diagnosis of GD in pregnancy should always be confirmed by measuring circulating free thyroid hormone levels (FT3 and FT4) and TSH. Since radioactive iodine scans are absolutely contraindicated in pregnancy, making a definitive diagnosis can be challenging. Presence of diffuse goitre, ophthalmopathy and hyperthyroid symptoms prior to pregnancy are some of the features that favour the diagnosis of GD. Presence of thyroid hormone receptor antibody (TRAb), although not routinely done in India, also confirms the diagnosis of Graves` disease. Ultrasound of the thyroid gland can be a practical alternative to thyroid scintigraphy in pregnant and lactating women .It is a non-invasive, portable, cost effective and safe imaging modality which will not only help in differential diagnosis of Graves' disease but will also pinpoint small underlying nodules. ${ }^{6}$

\section{HCG mediated hyperthyroidism}

Serum Human Chorionic gonadotropin levels rise immediately after fertilization and peaks at 10-12 weeks of gestation, after which the levels tends to fall. The $\beta$ subunit of HCG and TSH are identical and as a result of this HCG has weak thyroid stimulating activity and may cause sub clinical hyperthyroidism or mild overt hyperthyroidism during the period of highest serum HCG concentrations. Few examples of HCG mediated hyperthyroidism are

1. Gestational transient thyrotoxicosis

2. Hyperemesis gravidarum

3. Trophoblastic hyperthyroidism.

\section{Gestational transient thyrotoxicosis}

At $10-12$ weeks of gestation which is the time for peak rise in serum HCG concentrations, serum total $\mathrm{T} 4$ and $\mathrm{T} 3$ concentrations increase and TSH is reduced. Thus in some women high HCG can cause biochemical hyperthyroidism. This is a self-limiting condition and subsides by 14 -18 weeks of gestation when HCG levels tend to fall. This phenomenon is termed as Gestational transient thyrotoxicosis.

\section{Management of gestational transient thyrotoxicosis}

Generally, gestational transient thyrotoxicosis does not require medication, unless if hyperemesis gravidarum is present, thus the patient has to be hospitalized to receive intravenous rehydration, electrolyte correction and antiemetic medication. ${ }^{7}$ Use of anti-thyroid drugs is not recommended in this situation because the free $\mathrm{T} 3$ and free T4 levels usually return to normal by about 20 weeks of gestation. However, in clinical situations where it is difficult to distinguish between Graves' disease and Gestational transient thyrotoxicosis and the patient is clinically toxic, a trial of Antithyroid medications can be started which can be withdrawn once the TSH reaches the euthyroid range.

\section{Hyperemesis gravidarum}

About $0.1 \%$ to $0.2 \%$ pregnant women suffer from severe nausea and vomiting associated with weight loss. This syndrome is termed as hyperemesis gravidarum. Sometimes the weight loss is more than $5 \%$ of the body weight. Hyperemesis gravidarum is sometimes associated with marked dehydration, ketonuria and electrolyte imbalance in form of hypokalaemia, hypernatremia and metabolic alkalosis. Hyperemesis gravidarum usually occurs in first trimester of pregnancy ${ }^{8}$. The thyroid hyper function in hyperemesis gravidarum usually does not require treatment with anti thyroid drugs because it is mild and subsides as serum HCG concentration falls.

\section{Trophoblastic hyperthyroidism: hydatidiform mole and choriocarcinoma}

Hyperthyroidism has been reported in patients with trophoblastic diseases both, Hydatidiform mole and Choriocarcinoma. Both the conditions are associated with high serum HCG concentrations. Several investigators have reported laboratory evidence of hyperthyroidism in women with molar pregnancy, with normalisation of thyroid function test following evacuation of the mole.

\section{Management of hyperthyroidism in pregnancy}

Antithyroid drugs are the mainstay of treatment in pregnancy. Carbimazole; its active metabolite methimazole and propylthiouracil have all been used for the treatment of hyperthyroidism in pregnancy. Radioactive iodine is absolutely contraindicated in pregnancy. Surgery is rarely indicated in pregnancy because it carries risk of spontaneous abortion and premature delivery. Subtotal thyroidectomy is indicated for patients who develop severe adverse effects to 
antithyroid drugs. The optimal time of surgery is in the second trimester. ${ }^{9}$

Role of beta adrenergic blocking drugs in controlling hypermatabolic symptoms in pregnancy is limited. They can only be used for a few weeks as their continued use can lead to intra uterine growth retardation. The prolonged use of beta blockers in pregnancy is also associated with the development of foetal apnoea and bradycardia. $^{10}$

Use of ATD in pregnancy is associated with adverse events. Cutaneous reactions, agranulocytosis and hepatotoxicity are the main effects observed with the use of ATD. Ai Yoshihara et al treated 91 newly detected pregnant women with Graves' disease with ATD (40 pts with MMI, 51 with PTU) and studied the adverse events. In this study the frequency of cutaneous reactions in patients treated with MMI was $12.5 \%$ compared to $5.9 \%$ in patients treated with PTU. None of the patient treated with MMI developed hepatotoxicity whereas $3.9 \%$ of the patients treated with PTU developed hepatic adverse events. $^{11}$

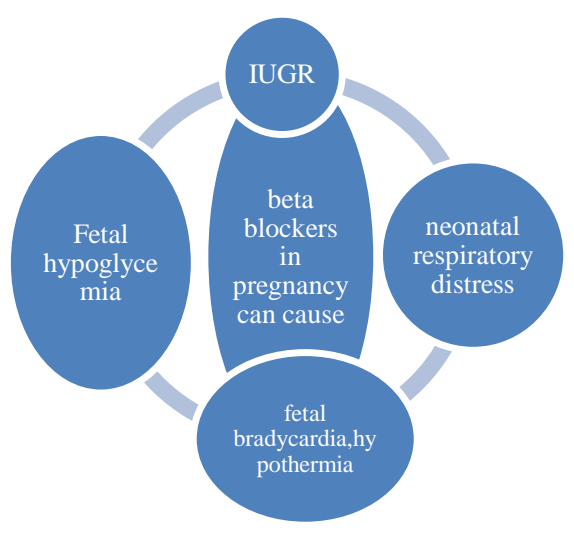

Figure 1: Effects of beta-blockers in pregnancy.

\section{Concerns with the use of antithyroid drugs in pregnancy}

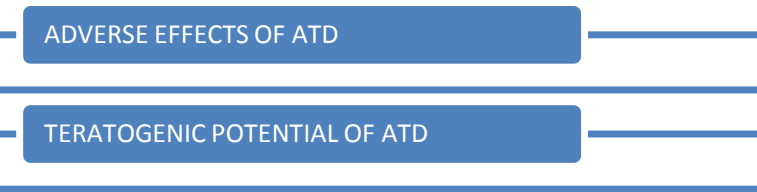

ATD AND RISK OF FETAL HYPOTHYROIDISM

Figure 2: Concerns with the use of anti-thyroid drugs in pregnancy.

\section{Adverse effects of ATD}

Antithyroid drugs are associated with development of both minor and major adverse effects. Minor side effects include cutaneous reactions (rash, urticaria) gastrointestinal upset, arthralgia (Table 2).

Among the major side effects, agranulocytosis is the most feared complication of ATD. Agranulocytosis is defined as an absolute granulocyte count less than 500 per cubic millimetre. In a large series of patients studied by Tajiri et al, agranulocytosis was reported in 0.37 percent of patients receiving propylthiouracil and in 0.35 percent of patients receiving methimazole. ${ }^{12}$

Table 2: Side effects of anti-thyroid drugs.

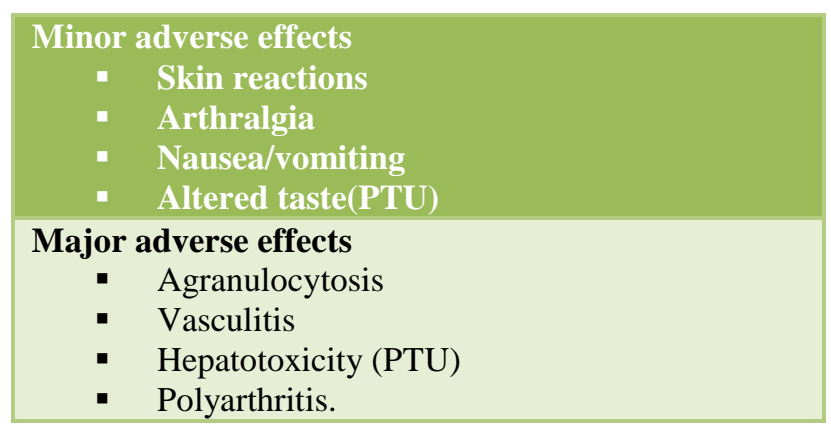

Hepatotoxicity is another major side effect of antithyroid medication. Immuno-allergic hepatitis occurs with the use of propylthiouracil. ${ }^{13}$

Vasculitis is the third major adverse event associated more often with the use of propylthiouracil than with carbimazole. Anti neutrophil cytoplasmic antibody positive vasculitis have been reported from Asian patients treated with propylthiouracil. ${ }^{13}$

\section{Teratogenic potential of ATD}

Medical literature is full of studies which show an association between the use of antithyroid medication for the treatment of hyperthyroidism in pregnancy and development of congenital malformations in the fetus. ${ }^{14}$ This association is widely reported with the use of carbimazole and its active metabolite methimazole. ${ }^{15}$ Carbimazole exposure in utero is associated with the development of birth defects like aplasia cutis, choanal atresia, tracheo-oesophageal fistula, patent vitellointestinal duct, and dysmorphic facies. These have been reported as components of carbimazole embryopathy. ${ }^{16}$

Many studies have confirmed that PTU does not represent a major teratogenic risk when used in clinically recommended doses in humans. ${ }^{17}$ Due to the concerns of teratogenicity associated with the use of methimazole during the first trimester of pregnancy; PTU is the preferred drug to be used for the treatment of hyperthyroidism in the first trimester. The American 
Thyroid Association recommends using PTU in the first trimester and switching to carbimazole /methimazole in the second trimester due to the fear of developing PTU related hepatotoxicity. It has been estimated that 4 out of approximately 4000 pregnant women treated with PTU in the US develop severe liver injury each year. ${ }^{18}$

\section{Antithyroid drugs and risk of foetal hypothyroidism}

Propylthiouracil and carbimazole /methimazole, both cross the placenta and are capable of causing foetal hypothyroidism and goitre. Hila Rosenfeld followed up 115 PTU exposed pregnancy and 1141 controls.Hypothyroidism was found in $9.5 \%$ of foetuses/neonates $(56.8 \%$ of whom had goitre). Hyperthyroidism possibly resulting from maternal disease was found in $10.7 \%$ foetuses. ${ }^{18}$

Polak et al studied 72 pregnant women with Graves's disease. Fetal ultrasonography was performed at 22 and 32 weeks of gestation age. Foetal goitre was found at 32 weeks in 11 of the foetuses of the 41 mothers with positive TSH-receptor antibodies and/or antithyroid treatment and in none of the fetuses of the 31 other mothers. In the 11 fetuses with goitre, ultrasound findings (thyroid Doppler and bone maturation), fetal heart rate, and maternal antibody and antithyroid drug status effectively discriminated between hypothyroidism $(n=7)$ and hyperthyroidism $(\mathrm{n}=4) .{ }^{19}$

Fetal goitre can be easily picked up by ultrasonography which should be done by an experienced ultrsonologist. This helps us to know the foetal thyroid status accurately so that timely dose interventions can be made in the drugs being offered to the mother. Close monitoring of thyroid function, roughly once a month, is important because the need for antithyroid treatment often declines through preg-nancy, and in the mid-trimester it may occasionally be discontinued. One should use the minimal dose of Antithyroid medication in order to keep the FT4 in the upper third of normal values for the prevention of neonatal hypothyroidism. ${ }^{18,19}$

\section{Foetal and neonatal hyperthyroidism}

Foetal and neonatal thyrotoxicosis is names given to the same disease manifesting at different periods of life. When it manifests in utero it is called fetal and when it manifests after the baby is born it is called neonatal thyrotoxicosis. Usually, foetal thyrotoxicosis continues as neonatal thyrotoxicosis after birth.

When patients with Grave's disease become pregnant, their thyroid stimulating antibodies can cross the placenta and stimulate the foetal thyroid triggering fetal thyrotoxicosis, which lasts until the maternal antibodies disappear from the fetal circulation. The prevalence of fetal thyrotoxicosis is low because pregnancy is a state of generalized immunosuppression and levels of thyroid receptor antibodies (TRAb) are reduced in pregnancy. It is only women who have three to five times normal levels of thyroid stimulating immunoglobulins (TSIs) result in foetal and neonatal thyrotoxicosis.

Pregnant women who were earlier treated for hyperthyroidism but are euthyroid at the time of current pregnancy can have high levels of TRAb in their sera, which is capable of causing foetal or neonatal thyrotoxicosis. Patients who have had radioiodine ablation are known to have persistent antibody levels. American thyroid association (ATA) recommends measurement of TRAb during 24-28 weeks of pregnancy and if the value is over three times normal, close follow for foetal thyrotoxicosis is recommended.

Foetal hyperthyroidism is a rare disease and occurs in 1 out of 70 pregnancies with Graves' disease or 1 in 400050,000 deliveries. It is characterized by foetal tachycardia, foetal goiter, fetal growth retardation and craniosyntosis. If left untreated, this disease can result in intrauterine death. ${ }^{19}$

Treatment of foetal hyperthyroidism consists of giving carbimazole to the mother which is then transferred through the placenta to the foetus .For management of neonatal hyperthyroidism carbimazole is given in the dose $0.5-1 \mathrm{mg} / \mathrm{Kg}$ daily. Propranolol $2 \mathrm{mg} / \mathrm{Kg}$ helps in slowing down the pulse rate of the neonate. Potassium iodide and glucocorticoids can also be given in severe cases. $^{20}$

\section{Neonatal hyperthyroidism}

Neonatal hyperthyroidism occurs due to persistence of maternal TSH receptor antibody (TRAb) and occurs in $1 \%$ of babies born to mothers with either active Graves' disease or those being previously treated for Graves' disease. ${ }^{21}$ Mothers with active Graves' disease or those who are TRAb positive after a definitive treatment in form of surgery or radioactive therapy should undergo evaluation of cord blood for measurement of neonatal free T4 and TSH levels. In addition to this, such infants should be closely observed for signs of thyrotoxicosis in the first few days of life.
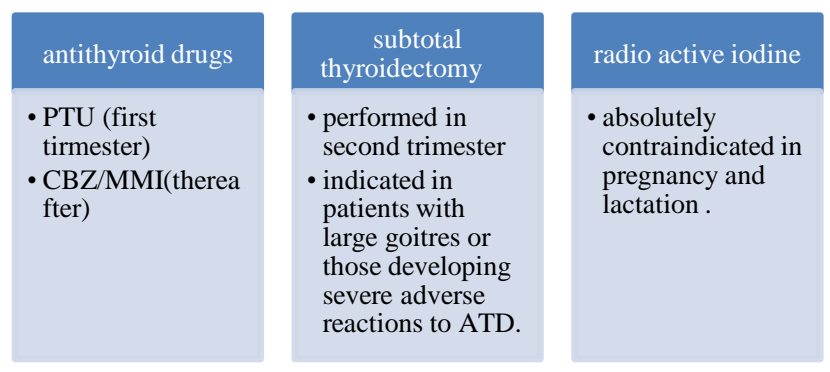

Figure 3: Treatment modalities: hyperthyroidism. 


\section{CONCLUSIONS}

Hyperthyroidism in pregnancy is associated with both maternal and foetal complications which can be avoided by rendering the patient euthyroid with the administration of anti-thyroid medications. Propylthiouracil and carbimazole/methimazole are the antithyroid drugs available with almost similar trans placental transfer rates. Use of carbimazole /methimazole in pregnancy is not only associated with increased incidence of scalp defect (aplasia cutis) in the infants, but some specific congenital malformation like choanal atresia, oesophageal atresia, trachea-oesophageal fistula, patent vitello intestinal duct, omphalocele ,dysmorphic facial features and growth retardation do occur. These malformations represent carbimazole/methimazole embryopathy. Due to the association of foetal teratogenicity with carbimazole/methimazole, propylthiouracil is recommended as the drug of choice in first trimester of pregnancy. However, as its use is associated with risk of hepatotoxicity, it should be changed to carbimazole/methimazole thereafter. Performing prenatal ultrasound directed to the foetal thyroid gland is of utmost importance as it enables prenatal diagnosis and treatment by dose modification in order to prevent foetal goitre. Methimazole/carbimazole remains the mainstay of treatment during the post-partum period when the mother is lactating.

Funding: No funding sources Conflict of interest: None declared

Ethical approval: Not required

\section{REFERENCES}

1. Glinoer D. Thyroid hyperfunction during pregnancy. Thyroid. 1998;8(9):859-64.

2. Cooper DS, Lauberg P. Hyperthyroidism in pregnancy. The Lancet Diabetes \&endocrinology. 2013;3:238-49.

3. Aggarwal N, Suri V, Singhla R, Chopra S, Shikha P, Bhansali A. Pregnancy outcome in hyperthyroidism case control study. Gynecol Obstet Invest, 2014.

4. Milliar LK, Wing DA, Leung AS, Koonings PP, Montoro MN, Mestman JH. Low birth weight and pre eclampsia in pregnancies complicated by hyperthyroidism. Obstet Gynecol. 1994;84(6):946-9.

5. Momotani N, Noh J, Oyanagi H, Ishikawa N. Anti thyroid drug therapy for Graves' disease during pregnancy: optimal regimen for foetal thyroid status. N Eng J Med. 1986;315(1):24-8.

6. Alzahrani AS,Ceresini G,Aldasougi SA. Role of ultrasonography in the differential diagnosis of thyrotoxicosis: a non invasive, cost effective and widely available but underutilized diagnostic tool. Endocr Pract. 2012;18:567-78.
7. Albar MT, Adam JM. Gestational transient thyrotoxicosis .Acta Med Indones. 2009;41:99-104.

8. Brodsky JB, Cohen EN, Brown BW, Wu ML, Whitcher C. Surgery during pregnancy and fetal outcome. American Journal of Obstetrics and Gynecology. 1980;138:1165-7.

9. Redmond GP. Propanolol and fetal growth monitoring. Semianars in Perinatology. 1982;6:1427.

10. Yoshimura Noh J, Watanabe N, Iwaku K, Kobayashi S, Suzuki M, Ohye H, et al. Frequency of Adverse Events of Antithyroid Drugs Administered during Pregnancy. 2014 Journal of Thyroid Research. doi.org/10.1155/2014/952352.

11. Tajiri J, Noguchi S. Antithyroid drug induced agranulocytosis: special reference to normal white blood cell count. Thyroid. 2004;14:459-62.

12. Liaw YF, Huang MJ, Fan KD. Hepatic injury during propylthiouracil therapy in patients with hyperthyroidism. A cohort study. Annals of Internal Medicine. 1993;118:424-8.

13. Guston LE, Steil J, Caterson RJ. Anti thyroid drugs and anti neutrophil cytoplasmic antibody positive vasculitis: a case report and review of literature. J Clin Endocrinol Metab. 1999;84:13-6.

14. Foulds N, Walpole I, Elmslie F, Mansour S. Carbimazole embryopathy: an emerging phenotype. American Journal of Medical Genetics. 2005;132(2):130-5.

15. Myers AK, Reardon W. Choanal atresia - a recurrent feature of foetal carbimazole syndrome. Clinical Otolaryngology. 2005;30:364-83.

16. Wing DA, Millar LK, Koonings PP, Montoro MN, Mestman JH. A comparison of propylthiouracil versus methimazole in the treatment of hyperthyroidism in pregnancy. Am J Obstet Gynecol. 1994;170:90-5.

17. Cooper DS, Rivkess SA. Putting Propylthiouracil in perspective. J Clin Endo Metab. 2009;6:1881-2.

18. Rosenfeld H, Asher Ornoy, Svetlana Shechtman. Pregnancy outcome, thyroid dysfunction and fetal goitre after in utero exposure to propylthiouracil: a controlled cohort study. $\mathrm{Br} \mathrm{J}$ Clin Pharmacol. 2009;68(4):609-17.

19. Batra CM. Fetal and neonatal thyrotoxicosis. Indian J Endocr Metab. 2013;17:50-4.

20. Radetti G, Zavallone A, Gentili L.Fetal and neonatal thyroid disorder. Minerva Pediatrica. 2002;54:383400.

21. Skuza KA. Prediction of neonatal hyperthyroidism in infants born to mothers with Graves'disease. J Pediatr. 1996;128:264-8.

Cite this article as: Aggarwal $\mathrm{R}$, Chugh $\mathrm{P}$.

Management of hyperthyroidism in pregnancy. Int $\mathbf{J}$ Reprod Contracept Obstet Gynecol 2016;5:1-5. 